\title{
Linfadenectomia laparoscópica em cadela com neoplasia mamária
}

\author{
Laparoscopic lymphadenectomy in a bitch with breast cancer
}

\author{
Fernando Wiecheteck de Souza ${ }^{\mathrm{I}^{*}}$ Maurício Veloso Brun ${ }^{\mathrm{I}}$ Andrigo Barboza de Nardi ${ }^{\mathrm{II}}$ \\ Rafael Ricardo Huppes ${ }^{\mathrm{III}}$ Carolina Quarterone ${ }^{\mathrm{IV}}$ Talita Mariana Morata Raposo ${ }^{\mathrm{III}}$ \\ Priscila Natasha Kasper ${ }^{\mathrm{V}}$ Marília Teresa de Oliveira ${ }^{\mathrm{I}}$ Rogério Luizari Guedes $^{\mathrm{VI}}$
}

\section{- NOTA -}

RESUMO

A linfadenectomia laparoscópica é realizada de forma rotineira na medicina, contudo é pouco utilizada na veterinária. Neste relato, um canino fêmea apresentando tumores na cadeia mamária esquerda (M4 e M5), foi submetido à aplicação intradérmica do azul de metileno estéril, ao redor do maior tumor (M5), buscando-se demarcação dos vasos linfáticos e linfonodos regionais. Após 15 minutos, iniciou-se a linfadenectomia abdominal videolaparoscópica na região inguinal esquerda, seguida da ovário-histerectomia (OVH) lapararoscópica com três portais. Realizou-se ainda mastectomia total unilateral esquerda. Pela histologia, obtiveram-se dois linfonodos abdominais livres de células tumorais. A paciente não apresentou recidiva em 60 dias.

Palavras-chave: laparoscopia, metástase, drenagem linfática, linfonodo sentinela, cães.

\section{ABSTRACT}

The laparoscopic lymphadenectomy is routinely performed in medicine, but not in veterinary medicine. This report describes a case of female canine with tumors in the left mammary chain (M4 and M5), which was subjected to intra-dermal application of sterile methylene blue, around the largest tumor (M5) which tried to demarcate the lymphatic vessels and regional lymph nodes. After 15 minutes, laparoscopic lymphadenectomy involving the abdominal in the left pelvic region started, followed by laparoscopic ovariohysterctomy $(\mathrm{OVH})$ using three portals access. It was also performed an unilateral left total mastectomy. For histology, two abdominal lymph nodes were free of tumor cells. The patient had no recurrence in 60 days.

Key words: laparoscopic, metastasis, lymphatic drainage, sentinel node, dogs.
As neoplasias mamárias em animais são tratadas cirurgicamente, devido à possibilidade de malignidade tumoral e de ocorrência de metástase subsequente para outros órgãos (SCHRENK et al., 2007). Nessas situações, o sistema linfático desempenha papel importante, tanto no controle local tumoral como no mecanismo de transporte celular, sendo fator associado ao aparecimento de metástases em diversos tipos de neoplasia (HAIGH \& GIULIANO, 2000). A formação de novos vasos linfáticos, decorrente de fatores linfangiogênicos, relaciona-se também com o período de desenvolvimento da tumorigênese.

Existem alguns trabalhos que demonstram, através da impregnação de marcadores, o sistema linfático regional associado (PINHEIRO et al. 2003; SUGA et al., 2007; PINHEIRO et al., 2009; MIYASHIRO et al., 2010). Foram testados diferentes tipos de marcadores, sendo o azul de metileno estéril o mais comumente utilizado (PINHEIRO et al., 2003). Outros mais recentemente testados são o iopamidol (SUGA et al., 2007), a hemossiderina (PINHEIRO et al., 2009) e o verde de indocianina (MIYASHIRO et al., 2010). A marcação linfática torna-se importante em casos com neoplasias mamárias em cadelas, visando a verificar

'Programa de Pós-graduação em Cirurgia Veterinária, Universidade Federal de Santa Maria (UFSM), Santa Maria, RS, Brasil. E-mail: wiecheteck@ hotmail.com. *Autor para correspondência.

"Programa de Pós-graduação em Clínica Cirúrgica, Universidade Estadual Júlio de Mesquita Filho (UNESP), Jaboticabal, SP, Brasil.

IIIPrograma de Pós-graduação em Clínica Médica, UNESP, Jaboticabal, SP, Brasil.

${ }^{\text {IV }}$ Programa de Pós-graduação em Anestesiologia Veterinária, UNESP, Botucatu, SP, Brasil.

vPrograma de Residência Médico Veterinária, Universidade Federal do Rio Grande do Sul (UFRGS), Porto Alegre, RS, Brasil.

${ }^{\mathrm{VI} M e ́ d i c o ~ V e t e r i n a ́ r i o ~ A u t o ̂ n o m o, ~ C u r i t i b a, ~ P R, ~ B r a s i l . ~}$ 
o aparecimento de metástases em tecidos celulares e delimitar os vasos linfáticos e linfonodos regionais, facilitando assim as suas extirpações. Estudando-se o sistema linfático mamário em cadelas saudáveis e com neoplasia, com o emprego de azul de metileno estéril (intradérmico, sob o parênquima mamário), foi demonstrada neoformação vascular e linfática nessa espécie (PINHEIRO et al., 2003).

A linfadenectomia laparoscópica é uma técnica que vem sendo utilizada com frequência, na medicina, em pacientes com neoplasias malignas, nos quais os tumores são grandes e existe o envolvimento do linfonodo alvo (LAVERY et al., 2010). Assim, a laparoscopia possui papel prognóstico, utilizando-se da biópsia para determinação da evolução da doença, e também terapêutico, quando é realizada a retirada cirúrgica dos linfonodos e vasos linfáticos envolvidos (LAVERY et al., 2010). Há ainda a possibilidade de se realizar uma laparoscopia exploratória na cavidade abdominal, auxiliando também no diagnóstico de outros possíveis focos tumorais (FREEMAN \& POTTER, 1999).

Um canino fêmea, SRD, com $12 \mathrm{~kg}, 9$ anos, apresentando tumores na cadeia mamária esquerda (glândula mamária abdominal medial "M4" e caudal "M5"), foi atendida e encaminhada para realização da mastectomia radical unilateral esquerda. Foi coletada amostra de sangue para realização de hemograma completo e funções renal (ureia e creatinina) e hepática (ALT e AST), e os valores encontravam-se dentro dos fisiológicos para a espécie.

A medicação pré-anestésica constou de acepromazina $0,2 \%\left(0,05 \mathrm{mg} \mathrm{kg} \mathrm{kg}^{-1}, \mathrm{IM}\right)$ associada ao tramadol (3mg kg-1, IM). Administrou-se cefazolina sódica a $20 \%\left(20 \mathrm{mg} \mathrm{kg}^{-1}\right.$, IV) e, após 30 minutos, realizou-se a indução anestésica, utilizando o propofol (5mg kg $\left.{ }^{-1}, \mathrm{IV}\right)$, seguido da entubação orotraqueal. A manutenção anestésica foi realizada com isofluorano $1 \%$ em oxigênio a $100 \%$ por meio de vaporizador universal em um fluxo de $0,08 \mathrm{~L} \mathrm{~min}^{-1}$. Administrouse fluidoterapia com $\mathrm{NaCl}$ a $0,9 \%\left(10 \mathrm{~mL} \mathrm{~kg}^{-1} \mathrm{~h}^{-1}\right)$. $\mathrm{O}$ animal foi então posicionado em Trendelenburg de aproximadamente $30^{\circ}$. Foi aplicado $1 \mathrm{~mL}$ do azul de metileno estéril (Figura 1A) em três pontos ao redor da mama abdominal caudal esquerda, e então masageou-se por 2 minutos a região para facilitar a drenagem do marcador linfático. Após 15 minutos da aplicação do marcador linfático (Figura 1B), iniciouse o procedimento videolaparoscópico.

$\mathrm{Na}$ região pré-umbilical da linha média ventral, à distância de $1 \mathrm{~cm}$ da cicatriz umbilical, realizou-se incisãode pele de aproximadamente $11 \mathrm{~mm}$. Pela técnica aberta, foi inserido o primeiro trocarte (10mm), obtendo-se a insuflação de $\mathrm{CO}_{2}$ medicinal (1L $\min ^{-1}$ ) até alcançar pressão intracavitária de $12 \mathrm{mmHg}$. Sob visualização endoscópica (endoscópio de $0^{\circ}$ e $10 \mathrm{~mm}$ ), elegeram-se as duas outras posições para introdução dos outros portais. A segunda punção foi realizada na parede abdominal esquerda $(5 \mathrm{~mm})$, enquanto o terceiro acesso foi obtido na parede abdominal direita (10mm). Realizou-se a inspeção da cavidade abdominal, iniciando-se a linfadenectomia da região púbica esquerda, abrangendo a área do anel inguinal interno até os vasos epigástricos profundos. $\mathrm{O}$ tecido adiposo envolvendo o trajeto linfático foi dissecado e extirpado com pinça Kelly e tesoura Metzenbaum, sendo retirados da cavidade pelo portal de $10 \mathrm{~mm}$, com o auxílio de saco de coleta, obtido com dedo de luva cirúrgica. Após a verificação de ausência de hemorragia, partiu-se para OVH laparoscópica, utilizando os mesmos portais de acesso.

A OVH iniciou com a secção do ligamento suspensor esquerdo, seguida da cauterização bipolar, abrangendo o mesovário e o complexo arteriovenoso ovariano (CAVO). Promoveu-se a secção dessas estruturas com tesoura de Metzenbaum, incluindo o mesométrio e o ligamento redondo. As mesmas etapas foram repetidas no pedículo ovariano direito. Cranialmente a cérvix, realizou-se a oclusão dos vasos uterinos, utilizando quatro clipes de titânio tamanho médio. Seccionou-se então essa estrutura na porção mais cranial a cérvix, utilizando tesoura de Metzenbaum e, em seguida, o útero e os ovários foram removidos pelo trocarte $(10 \mathrm{~mm})$. Verificouse a ausência de hemorragias, sendo a cavidade desinsuflada e as feridas de acesso suturadas. Empregou-se padrão Sultan na musculatura com fio poligalactina 910 2-0.

Após esse segundo procedimento laparoscópico, partiu-se para realização da mastectomia total unilateral esquerda pela técnica aberta. Foi realizada incisão elíptica na pele, ao redor da cadeia mamária, adotando-se margem em relação aos tumores de cerca de $2 \mathrm{~cm}$, aproveitandose ainda a área das feridas utilizadas para o primeiro e segundo portais. Em seguida, foi aplicada tração manual, com o auxílio de uma compressa úmida, sob o segmento cutâneo incisado, promovendo a ressecção do coxim gorduroso, dos linfonodos inguinais, glândulas mamárias e tumores. A hemorragia superficial foi controlada com o auxílio de eletrocoagulação monopolar. Isolaram-se os vasos epigástricos superficiais e caudais, e foram aplicadas duas ligaduras com poligalactina 910 2-0. O tecido subcutâneo foi ocluído em padrão "walking suture" e a pele em padrão interrompido simples com náilon monofilamentar 4-0. 


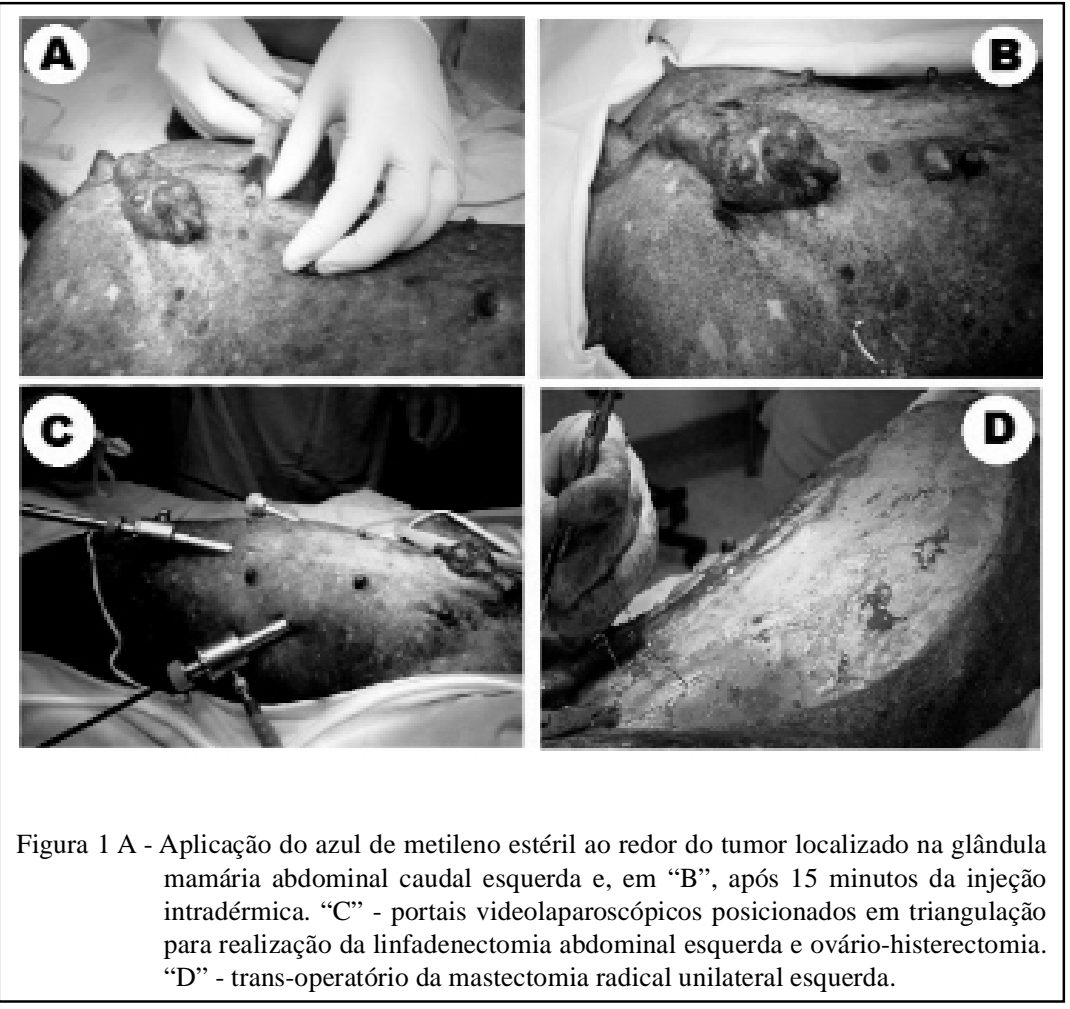

Após a recuperação anestésica, foi administrado meloxicam $\left(0,2 \mathrm{mg} \mathrm{kg}^{-1}\right.$, IM, s.i.d, por três dias) e tramadol ( $3 \mathrm{mg} \mathrm{kg}^{-1}, \mathrm{SC}$, t.i.d, por 5 dias). O curativo foi obtido com micropore após instilação de Rifamicina $\left(10 \mathrm{mg} \mathrm{mL}^{-1}\right)$. O animal recebeu a alta hospitalar em dois dias e, ao final de 10 dias, demonstrava cicatrização em primeira intenção. Após 60 dias, não se verificou recidiva da neoplasia. Os procedimentos duraram 45, 35 e 150min, respectivamente.

Este relato reforça o uso do azul de metileno como marcador linfático em procedimentos cirúrgicos que envolvem neoplasmas mamários, sem danos aparentes ao animal, apesar de PINHEIRO et al. (2009) afirmarem possíveis complicações que possam ocorrer com sua utilização, como um caso de hipersensibilidade severa no local da aplicação, fato este não observado no presente relato. Uma opção para substituição desse corante sintético seria o uso da hemossiderina (sangue hemolisado), conforme demonstrado no mesmo estudo, que obteve êxito em $100 \%$ dos animais, ao comparar com o resultado positivo envolvendo $85 \%$ do grupo que utilizou o azul de metileno estéril. Outros marcadores que também poderiam ser estudados e utilizados, porém seu uso ainda é limitado, é o iopamidol que, segundo SUGA et al. (2007), é identificado somente através da tomografia computadorizada, e o verde de indocianina, que, ainda em fase experimental, demonstrou resultados favoráveis em linfadenectomia laparoscópica da região pélvica MIYASHIRO et al. (2010).

O tempo de impregnação linfática de aguardado para coleta foi 60 minutos. Nos únicos dois microlinfonodos que foram coletados junto à gordura adjacente aos vasos ilíacos, não se constatou a presença de azul de metileno, somente sendo observado o corante junto ao tecido neoplásico extirpado e linfonodo inguinal, conforme PINHEIRO et al. (2003) também constatou. Estudos futuros poderão ser realizados utilizando tempos diferentes de impregnação intradérmica com azul de metileno ou outros marcadores linfáticos, buscando-se melhores ferramentas para o estadiamento de neoplasia mamária em cadelas.

A linfadenectomia abdominal é uma técnica que vem sendo executada nos casos de neoplasias de vesícula urinária em humanos, em que a cistectomia geralmente também é recomenda, conforme LAVERY et al. (2010). No presente relato, a linfadenectomia foi realizada associada à presença de neoplasias mamárias em uma cadela, permitindo a coleta de dois microlinfonodos, os quais não 
apresentavam alterações estruturais ou presença de células tumorais. Com o presente, comprova-se que a linfadenectomia abdominal em cadelas é viável por laparoscopia, além de haver a possibilidade de aplica-lá em animais com neoplasia mamária para estadiamento tumoral. No conhecimento dos autores, essa proposta ainda não havia sido trazida para a rotina cirúrgica de pequenos animais.

\section{COMITÊ DE ÉTICA E BIOSSEGURANÇA}

Declaração dos autores: O relato foi executado com animal atendido na rotina clínica do Hospital Veterinário da Universidade de Franca - SP e não foi parte de qualquer projeto, sendo apenas um relato de caso clínico, então os autores ficam a disposição para quaiquer esclarecimentos futuros sobre o mesmo.

\section{REFERÊNCIAS}

SCHRENK, P. et al. Morbidity following sentinel lymph node biopsy versus axilary lymph node dissection for patients with breast carcinoma. Cancer, v.88, p.608-614, 2000. Disponível em: <http://onlinelibrary.wiley.com/doi/10.1002/(SICI) 10 970142(20000201)88:3\%3C608::AIDCNCR17\%3E3.0.CO;2-K/ full>. Acesso em: 30 maio, 2012. doi: 0.1002/(SICI)10970142(20000201)88.

HAIGH, P.I.; GIULIANO, A.E. Role of sentinel lymph node dissection in breast cancer. Annals of Medicine, v.32, p.51-56, 2000. Disponível em: <http://informahealthcare.com/doi/abs/1 0.3109/07853890008995910>. Acesso em: 30 maio, 2012. doi: $10.3109 / 07853890008995910$.
PINHEIRO, L.G.P. et. al. Estudo experimental de linfonodo sentinela na mama da cadela com azul patente e tecnécio Tc99. Acta Cirúrgica Brasileira, v.18, p.545-552, 2003. Disponível em: 〈http://www.scielo.br/pdf/acb/v18n6/a12v18n6.pdf>. Acesso em: 30 maio, 2012 .

SUGA, K. et al. Cutaneous drainage lymphatic map with interstitial multidetector-row computed tomographic lymphography using iopamidol: preliminary results. Lymphology, v.40, p.6373, 2007. Disponível em: <http://www.ncbi.nlm.nih.gov/ pubmed/17853616>. Acesso em: 30 maio, 2012.

PINHEIRO, L.G.P. et al. Hemosiderin: a new marker for sentinel lymph node identification. Acta Cirúrgica Brasileira, v.24, p.432-436, 2009. Disponível em: <http://www.scielo.br/scielo. php?script=sci_arttext\&pid=S0102-86502009000600002 >. Acesso em: 30 maio, 2012. doi: 10.1590/S0102-865020009000600002.

MIYASHIRO, I. et al. Laparoscopic detection of sentinel node in gastric cancer surgery by indocyanine green fluorescence imaging. Surgical Endoscopy, v.25, n.5, p.1672-1676, 2011. Disponível em: 〈http://www.ncbi.nlm.nih.gov/pubmed/20976497〉. Acesso em: 30 maio, 2012. doi: 10.1007/s00464-010-1405-3.

LAVERY, H.J. et al. Robotic extended pelvic lymphadenectomy for bladder cancer with increased nodal yield. British Journal of Urology, v.107, p.1802-1805, 2010. Disponível em: <http:// www.ncbi.nlm.nih.gov/pubmed?term=Robotic\%20extended $\% 20$ pelvic $\% 201$ ymphadenectomy $\% 20$ for $\% 20$ bladder $\% 20$ cancer $\% 20$ with $\% 20$ increased $\% 20$ nodal $\% 20$ yield $>$. Acesso em: 30 maio, 2012. doi:10.1111/j.1464-410X.2010.09789.x.

FREEMAN, L.J. Minimally Invasive Surgery of the hemolymphatic system. In: FREEMAN L.J.; POTTER, L. Veterinary endosurgery. St Louis:Mosby, 1999. p.192-204. 